\title{
Work engagement y su relación con la performance y la satisfacción laboral
}

\author{
María Laura Lupano Perugini ${ }^{1}$ y Santiago Waisman ${ }^{2}$
}

\begin{abstract}
RESUMEN
El presente trabajo, basado en los aportes de la Psicología Organizacional Positiva (POP), consistió en un análisis del concepto de Work Engagement. Por un lado, se buscó explorar las concepciones que tienen personas con personal a cargo sobre el work engagement mediante entrevistas realizadas a 38 voluntarios de la población general de la Ciudad de Buenos Aires y el conurbano bonaerense (18 hombres, 47.37\%; 20 mujeres, 52.63\%). La edad promedio fue de 49.87 años $(D E=9.48)$. Del análisis de contenido realizado se obtuvieron una serie de categorías que reflejan concepciones acerca del engagement. Por ejemplo, los participantes consideraron que las personas con engagement son aquellas que muestran dedicación, motivación y son responsables. A su vez, se buscó evaluar la relación que presenta el engagement con la performance y la satisfacción laboral, así como examinar diferencias según género, edad y antigüedad laboral. La segunda muestra constó de 100 voluntarios de población general (52 hombres, 52\%; 48 mujeres, 48\%), que trabajan como empleados en organizaciones públicas y privadas de la Ciudad de Buenos Aires y el conurbano bonaerense. La edad promedio fue de 31.82 años $(D E=10.18)$. Se aplicó la Escala de Compromiso con el Trabajo (ECT) y escalas diseñadas ad hoc para evaluar performance laboral (individual y organizacional) y satisfacción laboral. Los resultados mostraron asociaciones positivas entre las tres dimensiones de work engagement (atención, absorción y energia) y la performance laboral (individual y organizacional) así como una asociación positiva entre las dimensiones de atención y energía con la satisfacción laboral. Asimismo, se obtuvieron diferencias según género en las dimensiones absorción y energía a favor de las mujeres. Dichos resultados son de fundamental importancia en el campo aplicado, dada la asociación de la variable psicológica estudiada -engagement- con la obtención de óptimos resultados en el terreno laboral.

Palabras clave: work engagement, satisfacción laboral, performance laboral, género, edad.
\end{abstract}

\section{Work engagement and its relation to performance and job satisfaction}

\begin{abstract}
The current work, based on the contributions of Positive Organizational Psychology, analyzed the concept of Work Engagement. On one side, the work aimed to explore the conceptions that people, who occupy a leader position, have about work engagement, through interviews which were carried out with 38 volunteers of the general population of the city of Buenos Aires and surroundings (18 males, 47.37\%; 20 females, 52.63\%). The mean age was 49.87 years old $(D E=9.48)$. According to the content analysis conducted, a series of categories were obtained that reflect conceptions about engagement. Moreover, the research analyzed the relationship between engagement, work performance and job satisfaction. It was used the Escala de Compromiso con el Trabajo (ECT) and some scales were designed ad hoc to assess job performance -at individual and organizational level-
\end{abstract}

\footnotetext{
${ }^{1}$ Consejo Nacional de Investigaciones Científicas y Técnicas (CONICET), Universidad de Palermo, Argentina; mllupano@hotmail.com

2 Universidad de Palermo, Argentina.
} 
and job satisfaction. The second sample consisted of 100 volunteers of the general population (52 males, 52\%; 48 females, 48\%) who worked as employees in public and private organizations in the city of Buenos Aires. The mean age was 31.82 years old $(D E=$ 10.18). The results showed positive associations among the three dimensions of work engagement and job performance (individual and organizational) as well as a positive association between the attention and energy dimensions and job satisfaction. In addition, women obtained higher scores on absorption and energy. These results are important in the applied labor field given the association of the psychological variable studied -engagement- with effective results.

Keywords: work engagement, job satisfaction, work performance, gender, age.

Durante mucho tiempo, las concepciones tradicionales sobre organizaciones han trasmitido una mirada que concibe a las mismas como caracterizadas por la avaricia y el egoísmo, y donde la creación de riqueza es la única medida de éxito. Esta concepción deja de lado aspectos que, en una época como la actual, son fundamentales para una comprensión adecuada del fenómeno abordado. Una nueva mirada sobre las organizaciones permite verlas como lugares donde la colaboración, la apreciación y el significado están presentes regularmente, donde el bienestar humano se vuelve un indicador clave del éxito y las relaciones sociales se caracterizan por la lealtad, la compasión, el respeto y la honestidad (Cameron \& Spreitzer, 2012).

La Psicología Organizacional Positiva (POP) -creada en el marco de la Psicología Positiva (Seligman, 1999) - se adscribe a esta última concepción, y desde ella trata de estudiar fenómenos que antes no habían sido abordados con el adecuado hincapié, tales como el engagement, la satisfacción laboral o el compromiso de los miembros con las organizaciones, entre otros. Esto permite sostener la conclusión de que una organización no es positiva solo por los resultados obtenidos, sino cuando logra incrementar el bienestar de sus miembros (Keyes, Hyson, \& Lupo, 2000).

Uno de los conceptos fundamentales de esta perspectiva es el de work engagement -compromiso con el trabajo-, entendido como la presencia psicológica en un rol, la atención de la persona, su absorción y la energía dirigida hacia tareas relacionadas al trabajo (Cameron \& Spreitzer, 2012). Las investigaciones de Kahn $(1990,1992)$ sirvieron de base para el desarrollo del concepto como un estado caracterizado por la presencia psicológica del individuo, el cual tiene importantes implicaciones para el propio éxito, así como para el de la organización.

Posteriormente, diversos estudios han analizado la composición del constructo (e.g., Rich, LePine, \& Crawford, 2010; Rothbard, 2001; Rothbard \& Patil, 2012; Schaufeli \& Bakker, 2004), para llegar a la conclusión de que está compuesto por múltiples factores.

El problema de la definición del término ha sido muy discutido en el campo de la POP (Bakker, Schaufeli, \& Leiter, 2008), lo que llevó a diferentes concepciones de lo que este significa. Una de las primeras definiciones lo ubica como un constructo opuesto al burnout, en cuanto este último constituye el extremo negativo de un continuum que va desde el cansancio, el desgaste, el cinismo, la despersonalización en el trabajo, hacia la energía, el compromiso, el involucramiento, el sentido de pertenencia (Maslach, Schaufeli, \& Leiter, 2001). Schaufeli y Bakker (2004) diferenciaron entonces tres dimensiones: vigor -que refiere a altos niveles de energía mental y 
resiliencia-, dedicación - que hace referencia a ser desafiado e inspirado por el trabajo- y absorción - que consiste en concentrarse totalmente en el propio trabajo-. Muchos estudios han utilizado esta caracterización, pero otros han criticado la dependencia teórica del concepto de burnout, indicando que algunas de las dimensiones propuestas por Schaufeli y Bakker se superponen con constructos ya existentes, como afecto positivo (Zhang, Rich, \& LePine, 2009)

Rich et al. (2010) retomaron el concepto de Kahn $(1990,1992)$ y Rothbard (2001) al plantear tres componentes: uno físico -que refiere a la energía física volcada al trabajo-, uno emocional -que involucra una sensación placentera y la activación de afecto positivo- y uno cognitivo -que refiere a la absorción y la atención-. Esta definición también cuenta con algunos problemas, como la dificultad de separar el componente emocional del constructo afecto positivo, o el hecho de que se une absorción y atención en una dimensión, cuando la evidencia sugiere que son dimensiones separadas (Rothbard, 2001).

Teniendo en cuenta estos antecedentes, se considerarán las dimensiones propuestas por Rothbard y Patil (2012): atención -que refiere a los recursos que una persona puede aplicar a una tarea determinada-, absorción -que refiere a la capacidad de aplicar esos recursos con intensidad- y energía-que consiste en un componente fisico que puede ser dirigido a la tarea-. Nienaber y Martins (2014) señalan que, a pesar de las diferencias detalladas en cuanto a su definición, se han diseñado varios instrumentos para medir work engagement. Para el presente estudio se utiliza una escala diseñada en Argentina (Lupano Perugini, de la Iglesia, Castro Solano, \& Fernández Liporace, 2017) sobre la base de esta última conceptualización.

El engagement cobra especial relevancia en el ámbito laboral, dada su asociación con variables de resultado, por lo que variadas investigaciones lo relacionan tanto con altos índices de satisfacción como de performance laboral. Por ejemplo, muchos estudios han encontrado que está fuertemente asociado a la satisfacción laboral al dar cuenta de que el estado psicológico de presencia en un rol facilita la sensación de bienestar en el trabajo (e.g., Alarcon \& Edwards, 2011; Høigaard, Giske, \& Sundsli, 2012; Karatepe \& Karadas, 2015; Mache, Vitzthum, Klapp, \& Danzer, 2013). Sin embargo, existen pocos estudios a nivel latinoamericano, en especial en el contexto argentino. La satisfacción laboral es una actitud que las personas tienen hacia su trabajo y que engloba diferentes facetas (e.g., satisfacción con el supervisor, con los compañeros de trabajo, con la remuneración, con las posibilidades de ascender, así como con el trabajo en general) (Cameron \& Spreitzer, 2012; Spector, 1997). Por otro lado, múltiples estudios han encontrado asociación entre el work engagement y la performance laboral que alude a cuán bien se desenvuelve un individuo en las tareas necesarias para un rol laboral y a cómo contribuye positivamente al contexto social y psicológico de una organización (Christian, Garza, \& Stalker, 2011). De acuerdo con Christian et al. (2011), un empleado con alto engagement estaría motivado para llevar las tareas de su rol con más persistencia, intensidad y concentración. En relación con lo antedicho, Albrecht, Bakker, Gruman, Macey y Saks (2015) argumentan que la politica de Recursos Humanos debe fomentar los niveles de engagement, ya que esto repercute en los resultados de la organización, sobre todo si se trabaja en los aspectos vinculados con la energía (Owens, Baker, Sumpter, \& Cameron, 2016). 
Por otro lado, estudios previos han investigado las diferencias en el nivel de engagement según sexo, en donde se encontraron resultados contradictorios. En un estudio sobre una muestra de 25.000 empleados de 13 países diferentes (Australia, Bélgica, Canadá, Finlandia, Francia, Alemania, Grecia, Holanda, Noruega, Portugal, España, Sudáfrica y Suecia) se hallaron niveles levemente superiores en los hombres, aunque los autores aclaran que las diferencias son pequeñas y con poca relevancia práctica (Salanova \& Schaufeli, 2004). Por su parte, Mastenbroek et al. (2015) analizaron una muestra de profesionales holandeses, en donde encontraron también una leve pero significativa diferencia a favor de los hombres. Sin embargo, Mauno, Kinnunen y Ruokolainen (2007) hallaron resultados opuestos a los anteriores en un estudio realizado sobre una muestra de profesionales de la salud en Finlandia, ya que las mujeres presentaron niveles levemente más altos de engagement. Por último, en un estudio más reciente, Liu, Cho y Putra (2017) no hallaron diferencias significativas en los niveles de engagement según sexo. Estos antecedentes dan cuenta de que probablemente incidan factores culturales en los resultados hallados.

Otra variable cuyo efecto sobre el engagement ha sido estudiado es la antigüedad en la organización, también llamada tenure en inglés. Ho, Wong y Lee (2011) hallaron una asociación positiva entre estas variables, al igual que Cahill, McNamara, Pitt-Catsouphes y Valcour (2015), quienes también encontraron que quienes tenían más antigüedad presentaban niveles más altos de work engagement. Sin embargo, otros estudios no encontraron una asociación significativa entre estas variables (e.g., Mattthijs Bal, De Cooman, \& Mol, 2013; Xu \& Cooper Thomas, 2010).

Por último, diversos antecedentes han investigado la relación entre edad y engagement. Algunas investigaciones han encontrado una asociación positiva entre estas variables, dando cuenta de que las personas de mayor edad se sienten más comprometidas o engaged al momento de trabajar (e.g., Owens et al. 2016; Schaufeli \& Bakker, 2004). No obstante, las relaciones encontradas son débiles en su mayoría, pues existen otros antecedentes que no han encontrado asociación entre estas variables (e.g., Lin et al., 2016).

La importancia del estudio que se presenta recae en analizar estos factores en el contexto latinoamericano, ya que la mayor parte de los estudios reportados son provenientes de otros entornos culturales (en especial, Estados Unidos y países de Europa). Por dicho motivo, también resulta relevante analizar las concepciones que trabajadores de Argentina tienen respecto del work engagement a fin de no imponer una noción que a nivel local no conserve el mismo sentido.

Sobre la base de los antecedentes teóricos y empíricos expuestos se plantean los siguientes objetivos:

- Explorar las concepciones que tienen empleados con personal a cargo acerca del work engagement y factores que lo favorecen.

- Determinar si existe relación entre work engagement, performance y satisfacción laboral.

- Determinar si existe relación entre el work engagement y determinadas variables individuales (e.g., edad de los empleados, antigüedad laboral, género). 


\section{MÉTODO}

Tipo de estudio y diseño: Estudio exploratorio, correlacional - de diferencias de grupos. Diseño transversal.

\section{Participantes}

En primer lugar, se realizaron entrevistas a 38 personas que trabajan con personal a cargo en organizaciones públicas y privadas de la Ciudad de Buenos Aires y el conurbano bonaerense. La edad promedio de las personas entrevistadas fue de 49.87 años $(D E=9.48)$. El $47.37 \%(n=18)$ de los participantes eran hombres, mientras que el $52.63 \%(n=20)$ eran mujeres. El $76.32 \%(n=29)$ residian en CABA y el $23.68 \%(n=9)$ lo hacian en GBA. De estos voluntarios, la antigüedad promedio en su organización fue de 16.61 años $(D E=12.90)$, con un máximo de 45 años y un mínimo de 1 año. El 15. $79 \%(n=6)$ trabajaban en organizaciones pequeñas, el $26.32 \%(n=10)$ en medianas y el $57.89 \%(n=22)$ en organizaciones grandes, de las cuales el 36. $84 \%(n=14)$ eran públicas y el $63.16 \%(n=24)$ privadas.

La segunda muestra empleada constó de 100 voluntarios de población general, que trabajan como empleados en organizaciones públicas y privadas de la Ciudad de Buenos Aires y el conurbano bonaerense, de entre 18 y 65 años. La edad promedio de los participantes fue de 31.82 años $(D E=10.18) \mathrm{y}$ con una antigüedad promedio en su organización de 5.77 años $(D E=7.00)$. E1 $52 \%(n=52)$ de los participantes fueron hombres y el $48 \%(n=48)$ mujeres. A su vez, el 83\% $(n=83)$ residia en la Ciudad autónoma de Buenos Aires CABA-, mientras que el $17 \%(n=17)$ lo hacía en el GBA. El $19 \%(n=19)$ de los voluntarios tenía estudios secundarios completos, el $74 \%(n=74)$ tenian estudios universitarios, y el $7 \%(n=7)$ tenía estudios de posgrado. Al considerar la clase socioeconómica, el $21 \%(n=21)$ se autopercibió como perteneciente a clase media alta, el $64 \%(n=64)$ a clase media y el $15 \%(n=$ 15) a clase media baja. Con respecto a las organizaciones en las que estos participantes trabajaban, el $17 \%(n=17)$ lo hacía en organizaciones de tamaño pequeño, el $15 \%(n=15)$ de tamaño medio y el $68 \%(n=68)$ de tamaño grande, siendo el $41 \%(n=41)$ públicas y el $59 \%(n=59)$ privadas. Las organizaciones pertenecian a diferentes rubros: salud, financiero, administración pública, comercial, entre otros. El $18 \%(n=18)$ de estos participantes tenía personal a cargo, mientras que el $82 \%(n=82)$ no tenía.

\section{Materiales}

En primer lugar, con la finalidad de responder al primer objetivo, se realizaron una serie de entrevistas estructuradas a empleados con personal a cargo. En estas entrevistas se les preguntó acerca de sus concepciones acerca del work engagement (i.e. conocimiento sobre el concepto, factores que favorecen el work engagement, características de una persona con alto nivel de engagement). A fin de contar con información adicional que permitiese una evaluación del fenómeno observado, se incluyó una escala de respuesta Likert con opción de 6 puntos $(1=$ Poco a $6=$ Mucho $)$ para que los entrevistados puntúen la performance, la satisfacción laboral y el nivel de engagement de los empleados a su cargo. Contaba, además, con un apartado que indagaba sobre el sexo, edad, lugar de residencia, lugar de nacimiento, estado civil, clase social, situación laboral, ocupación y nivel educativo de los participantes.

Escala de Compromiso con el Trabajo (ECT; Lupano Perugini et al., 2017). Se trata de una prueba especialmente diseñada para la evaluación del work 
engagement (compromiso con el trabajo) con base en la propuesta teórica de Rothbard y Patil (2012), que se refiere a un constructo multidimensional vinculado a la dedicación de un empleado a su rol y tareas laborales. Dicho constructo está compuesto por dos dimensiones cognitivas (atención y absorción) y una física (energía). Como resultado del proceso de validación, en Argentina se obtuvo una versión final de 11 items. Algunos ejemplos de ítems son los siguientes: "Concentro una gran cantidad de atención en mi trabajo" (Atención), "Cuando estoy trabajando, a menudo pierdo la noción del tiempo" (Absorción), "Dedico mucha energia a mi trabajo" (Energia). El formato de respuesta es tipo Likert de cinco puntos, con recorrido de 1 (completamente en desacuerdo) a 5 (totalmente de acuerdo). La prueba cuenta con evidencias de validez convergente y discriminante y se comprobó la estructura de tres factores mediante análisis factorial confirmatorio. Además, se estimó la consistencia interna para las tres dimensiones, y se obtuvieron valores adecuados (Atención $\alpha=.88$; Absorción $\alpha=.71$; Energía $\alpha=.80$ )

Además, se diseñaron una serie de protocolos para la evaluación de algunas de las variables consideradas en la investigación. Dichos protocolos fueron testeados previamente en un estudio piloto realizado con un grupo reducido de empleados $(n=15)$. Se modificaron algunas expresiones lingüisticas sugeridas por los participantes del estudio piloto, dando lugar a las versiones definitivas utilizadas. A continuación, se describen los protocolos diseñados:

Encuesta Sociodemográfica: Recaba datos personales de los participantes (sexo, edad, lugar de residencia, estado civil, nivel socioeconómico, nivel de estudios, ocupación, nivel socio - económico autopercibido).

Encuesta Organizacional: Consulta a los participantes sobre datos de la organización en la que trabajan y el puesto que ocupan (tamaño, tipo, rubro, área en la que trabaja, puesto y personal a cargo, antigüedad laboral).

Encuesta de Satisfacción Laboral: Se diseñaron seis ítems con opción Likert de respuesta que va de 1 (totalmente insatisfecho) a 7 (totalmente satisfecho) que evalúan cuán satisfecha se autopercibe la persona en cuanto a su trabajo en general y a diferentes aspectos de este (i.e., sueldo, jefes, compañeros, lugar, carrera). Un ejemplo de ítem es "¿Cuán satisfecho estoy con el sueldo que recibo?”. Para la elección de las áreas a evaluar (e.g., sueldo, jefes, compañeros) se tomaron en cuenta aspectos analizados en otros instrumentos previos (e.g., Balzer et al., 1997). Se calculó alfa de Cronbach para la escala total obteniéndose un valor aceptable de .79

Encuesta sobre Performance Organizacional e Individual: Frente a la imposibilidad de obtener indicadores objetivos sobre el rendimiento organizacional, se decidió inferirlo a partir de la percepción de los empleados. Se tomaron como indicadores de desempeño organizacional algunos utilizados en investigaciones internacionales sobre performance (e.g., Cameron, Brigth, \& Caza, 2004). Los mismos aluden a niveles de eficiencia, innovación, crecimiento, calidad, retención de empleados y clientes, satisfacción, adaptación. Por ejemplo, para el diseño de la primera sección de la encuesta orientada a la evaluación del desempeño organizacional- se construyeron diez items con opción Likert de respuesta -1 (Poco) a 6 (Mucho)-, considerando los indicadores de eficiencia propuestos por Cameron et al. (2004) (e.g., cumplimento de objetivos, atención a las demandas del cliente, utilización óptima de recursos, etc.). Un ejemplo de ítem es “ ¿En qué medida cree que la organización cumplió eficientemente -haciendo un buen uso de los recursos- 
con los objetivos propuestos?”. El alfa obtenido para este apartado fue de .86 . A partir del mismo criterio, se diseñó otra sección que solicitaba al participante que calificara su desempeño como empleado. Se diseñaron seis ítems con la misma opción de respuesta que el apartado anterior $(1=$ Poco y 6 = Mucho). Un ejemplo de ítem es: “¿En qué medida cree que los resultados obtenidos fueron de calidad?”. El alfa obtenido para esta sección fue de .85 .

\section{Procedimiento}

Los datos fueron recolectados por pasantes que se encontraban realizando una práctica de investigación en una universidad privada de la ciudad de Buenos Aires. Los participantes fueron voluntarios y no recibieron retribución alguna por su colaboración. Además, el cuadernillo que contenía las encuestas presentaba en su portada una introducción en la que se solicitaba el consentimiento del participante, se aseguraba el anonimato de los datos y su uso exclusivo para investigación. También se realizaron entrevistas por escrito a otra muestra de voluntarios de la población general que tenían personal a cargo. La recolección y carga de datos fue supervisada por un docente investigador.

\section{Análisis de datos}

En primer lugar, se realizó un análisis de contenido de las entrevistas realizadas, categorizando las respuestas dadas en las mismas. Luego, se realizaron pruebas de correlación bivariada $r$ de Pearson con el fin de analizar la asociación existente entre las distintas dimensiones de work engagement con la performance y satisfacción laboral, así como con la edad y la antigüedad laboral. Por último, se realizó una prueba $t$ de Student para evaluar las diferencias según género en el nivel de work engagement. E1 programa empleado para la carga y análisis de los datos fue SPPS 18.0.

\section{RESULTADOS}

Para responder al primer objetivo, se analizaron los datos de las entrevistas administradas a 38 personas con personal a cargo. En este objetivo se buscaba explorar las concepciones que presentan estos trabajadores acerca del work engagement. Frente a la pregunta acerca de si conocían el concepto, el $50 \%(n=19)$ de los participantes respondió que sí lo conocía y sus concepciones se correspondian con la definición del término, en tanto que el otro $50 \%(n=19)$ no estaba familiarizado con el mismo. A quienes no lo estaban se les brindó una breve explicación sobre el fenómeno.

Otro de los aspectos consultados fue acerca de los factores que consideraban que favorecen el work engagement en los empleados. A partir de las respuestas dadas se realizó un análisis de contenido de estas. Este análisis consiste en una técnica flexible que permite combinar categorias de un modelo propuesto con subcategorias que emanan de los datos analizados (Denecke \& Nedj1, 2009; Hsieh \& Shannon, 2005). Se lograron aislar ocho categorias a partir de 61 respuestas diferentes dadas por los participantes. Las más frecuentes fueron la presencia de desafios, el diálogo fluido y el reconocimiento (ver tabla 1). 
Tabla 1.

Factores que favorecen al work engagement.

\begin{tabular}{lcc}
\hline Categoría & Frecuencia & Porcentaje \\
\hline Desafíos & 15 & $23.08 \%$ \\
Diálogo Fluido & 15 & $23.08 \%$ \\
Reconocimiento & 15 & $23.08 \%$ \\
Reconocimiento Económico & 6 & $9.23 \%$ \\
Pasión & 4 & $6.15 \%$ \\
Sentido de Pertenencia & 4 & $6.15 \%$ \\
Clima Laboral & 2 & $3.08 \%$ \\
\hline
\end{tabular}

Por otro lado, se analizaron las respuestas dadas acerca de las características que debería tener una persona con alto nivel de work engagement. Se encontraron también ocho categorías (a partir de 71 respuestas diferentes dadas por los participantes). Las características más frecuentes se relacionan con ser dedicado, estar motivado y ser responsable (ver tabla 2).

Tabla 2.

Características de una persona con alto work engagement.

\begin{tabular}{lcc}
\hline Categoría & Frecuencia & Porcentaje \\
\hline Dedicado & 24 & $34.29 \%$ \\
Motivado & 19 & $27.14 \%$ \\
Responsable & 12 & $17.14 \%$ \\
Entusiasta & 5 & $7.14 \%$ \\
Formado & 4 & $5.71 \%$ \\
Apasionado & 3 & $4.29 \%$ \\
Flexible & 2 & $2.86 \%$ \\
Sin Categoría & 1 & $1.43 \%$ \\
\hline
\end{tabular}

A continuación, se trabajó con los datos de la segunda muestra de 100 empleados para responder al resto de los objetivos. En primer lugar, se estimaron los estadísticos descriptivos de las variables analizadas (work engagement, performance y satisfacción laboral). Los resultados muestran que los niveles de engagement de la muestra se encuentran levemente por encima del punto medio de la escala, lo que implica un nivel moderadamente bueno. Lo mismo se puede decir de la performance laboral, si bien hay que tener en cuenta que puede diferir de la evaluación de los supervisores. Por último, la muestra presenta un alto nivel de satisfacción laboral, muy cercano al máximo (ver tabla 3).

En este punto también pueden considerarse las evaluaciones que dieron los participantes con personal a cargo de la muestra analizada en el objetivo anterior. Estas personas debian puntuar en una escala Likert de 6 puntos los niveles de engagement, performance y satisfacción laboral que ellos consideraban que tenían sus empleados, dando como resultado un nivel promedio de engagement de $4.68(D E=0.96)$, de satisfacción laboral de 4.55 $(D E=0.89)$ y de performance laboral de $4.74(D E=0.98)$. Estos resultados son bastante similares a los hallados en la muestra de empleados.

Con la finalidad de responder al segundo objetivo, que intenta analizar la asociación que existe entre work engagement y performance laboral, se calcularon coeficientes de correlación bivariada $r$ de Pearson, y se obtuvo como resultado una asociación estadísticamente significativa y positiva entre la performance individual reportada y las dimensiones atención, absorción y 
energía, así como una asociación estadísticamente significativa y positiva entre estas tres dimensiones con la performance de la organización reportada. Con respecto a analizar la relación existente entre el work engagement y la satisfacción laboral, se obtuvieron resultados estadísticamente significativos para la dimensión atención y para la dimensión energía. Todas las correlaciones halladas presentan un tamaño del efecto pequeño $(r<.30)$ (Cohen, 1992). No se halló una asociación estadísticamente significativa con la dimensión absorción (ver tabla 3).

Tabla 3.

Estadísticos descriptivos y correlaciones entre las variables analizadas.

\begin{tabular}{lcccc}
\hline & \multicolumn{4}{c}{ Correlaciones } \\
\cline { 2 - 5 } & \multicolumn{1}{c}{$\boldsymbol{M ( D E )}$} & Atención & Absorción & Energía \\
\cline { 2 - 5 } Atención & $3.88(0.81)$ & - & - & - \\
Absorción & $3.42(0.71)$ & $.61^{* *}$ & - & - \\
Energía & $3.71(0.83)$ & $.68^{* *}$ & $.58^{* *}$ & - \\
Performance individual & $4.55(0.88)$ & $.21^{*}$ & $.24^{*}$ & $.32^{* *}$ \\
Performance organizacional & $3.99(0.89)$ & $.29^{* *}$ & $.24^{*}$ & $.23^{* *}$ \\
Satisfacción laboral & $5.11(1.13)$ & $.23^{*}$ & .18 & $.28^{* *}$ \\
\hline${ }^{* *} p<.01 ;{ }^{*} p<.05$ & & & &
\end{tabular}

En relación con el último objetivo, que pretende determinar si existe relación entre el work engagement y determinadas variables individuales, se obtuvieron los siguientes resultados. Analizando las diferencias en el nivel de engagement según género, se calculó una prueba $t$, a partir de la cual se encontró una diferencia estadísticamente significativa en las dimensiones absorción $[t=-3.75 ; 38 \mathrm{gl} ; p=.001]$ y energía $[t=-2.48 ; 38 \mathrm{gl} ; p=.015]$, en las que las mujeres mostraron niveles más altos tanto en absorción $\left[\mathrm{M}_{\text {mujeres }}=\right.$ 3.68 vs. $\left.M_{\text {hombres }}=3.18\right]$ como en energia $\left[M_{\text {mujeres }}=3.92\right.$ vs. $\left.M_{\text {hombres }}=3.51\right]$. No se encontraron diferencias estadísticamente significativas en la dimensión de atención según género, así como tampoco en la performance y satisfacción laboral. Además, tampoco se halló relación entre las dimensiones del work engagement y la edad de los trabajadores $(p>.05)$.

Por último, con referencia a la relación entre work engagement y antigüedad laboral se calcularon coeficientes de correlación bivariada $r$ de Pearson, pero no se encontró asociación estadísticamente significativa. Tampoco se hallaron correlaciones significativas en relación con la performance y satisfacción $(p>.05)$.

\section{CONCLUSIONES}

El presente estudio tuvo como primer objetivo explorar las concepciones acerca del work engagement en una muestra de 38 empleados con personal a cargo pertenecientes tanto al ámbito público como privado y de diferentes rubros laborales. Asimismo, se intentó determinar si existe relación entre el work engagement, la performance y la satisfacción laboral percibida, para la cual se trabajó con otra muestra de 100 empleados de organizaciones públicas $\mathrm{y}$ privadas de diferentes rubros, quienes pertenecian a diferentes edades y clases sociales.

En cuanto a los resultados obtenidos en el primer objetivo, resultó llamativo que la mitad de los participantes desconocieran el concepto tratado. De todos modos, al brindárseles la explicación sobre el mismo, la mayoría 
alegó que conocían de qué se trata, pero no lo identificaban con dicho nombre. Por otro lado, en relación con los factores que consideran que favorecen el engagement, así como las características que consideran que debe tener una persona con alto engagement, las categorias obtenidas resultan consistentes con las investigaciones previas sobre la temática (e.g., Rothbard \& Patil, 2012), ya que se relacionan tanto con el aspecto físico del engagement que es la energía (e.g., dedicado, entusiasta) como con los cognitivos, es decir, con la atención y la absorción (e.g., apasionado, motivado).

Los resultados hallados con respecto a la relación entre el work engagement y la performance laboral coinciden con estudios anteriores sobre la temática, los cuales encontraron una asociación similar (e.g., Alessandri, Borgogni, Schaufeli, Caprara, \& Consiglio, 2015; Bakker \& Matthijs Bal, 2010; Christian et al., 2011; Ho et al., 2011; Lin et al., 2016; Rich et al., 2010; Salanova \& Schaufeli, 2004). Tal como se ha estudiado en diferentes países, el hecho de que las personas estén absortas en su trabajo, que dediquen plenamente sus recursos atencionales a realizar sus tareas y que puedan emplear grandes cantidades de energía en las mismas lleva a que puedan superar las adversidades que enfrentan en su trabajo, y permitir que estén más preparados para sortear desafios y que realicen sus tareas de la mejor forma posible, lo cual se traduce en trabajadores más exitosos y efectivos, mejorando su performance y la de la organización a la que pertenecen.

Por otro lado, los resultados obtenidos en relación con la satisfacción laboral presentan cierta coincidencia con los antecedentes, los cuales también hallaron una asociación significativa y positiva entre el work engagement y la satisfacción (e.g., Alarcon \& Edwards, 2011; Alarcon \& Lyons, 2011; Ho et al., 2011; Høigaard et al., 2012; Salanova \& Schaufeli, 2004; Yakin \& Erdil, 2012). Esta asociación se debe a que las personas con alto engagement encuentran su trabajo como algo motivador, le dan un mayor significado personal, se sienten más conectados con su rol, presentan mayor entusiasmo y orgullo por los logros individuales y de la organización, lo cual se traduce en mayor satisfacción laboral (Yakin \& Erdil, 2012).

Las diferencias encontradas en el nivel de engagement según género coinciden con los resultados obtenidos por Mauno et al. (2007), aunque difieren de otros estudios anteriores, los cuales llegaron a resultados opuestos, o bien no encontraron ninguna diferencia significativa (e.g., Liu et al., 2017; Mastenbroek et al., 2015; Salanova \& Schaufeli, 2004). Vale aclarar que, en general, las diferencias halladas en muchos de los estudios citados fueron muy bajas y de poca relevancia práctica (Salanova \& Schaufeli, 2004), por lo que es posible que esta pequeña diferencia pueda explicarse por factores culturales. Una cuestión para tener en cuenta es que, en la muestra analizada en el presente estudio, las mujeres ocupaban cargos altos en la organización, lo cual puede estar ligado a un nivel de engagement más elevado que el de los hombres, que en su mayoría llevaban poco tiempo en su trabajo.

Por último, no se halló una asociación entre el work engagement tanto con la antigüedad laboral como con la edad de los trabajadores. Esto presenta cierta coincidencia con otros estudios (e.g., Mattthijs Bal et al., 2013; Xu \& Cooper Thomas, 2010; Lin et al., 2016). No obstante, los antecedentes suelen ser muy contradictorios y no muestran una tendencia uniforme (Goštautaite \& Bučiūnienè, 2017), lo cual haria pensar que son otras variables las que tendrian efecto sobre el work engagement. 


\section{Comentarios}

Estos hallazgos aportan información valiosa desde el marco de la POP en el contexto latinoamericano. Las organizaciones actuales cada vez dan más peso a conceptos como el work engagement o la satisfacción laboral, los cuales son esenciales para una buena performance individual y organizacional. Estudios como el presente dan apoyo a este cambio cultural dentro de las organizaciones y permiten que estas se enfoquen cada día más en mejorar el bienestar de sus empleados.

Además, el interés de los resultados obtenidos para el área aplicada se aprecia de modo inmediato. Las áreas de Personal o Recursos Humanos de las organizaciones podrían beneficiarse, entonces, reforzando los niveles de engagement de los empleados ya que aquí se ha verificado su relación con el rendimiento. Por lo tanto, deberian considerarse los factores que resultan importantes para aquellos que tienen personal a cargo, a fin de incrementar los niveles de engagement de sus empleados y trabajar sobre esos aspectos (e.g., presentar desafios, mantener un diálogo fluido, dar reconocimiento por las tareas, entre otros)

Si tenemos en cuenta las limitaciones de este estudio, primero debe considerarse que el tamaño de las muestras analizadas es reducido, lo que afecta la generalización de los hallazgos. Además, el empleo de medidas subjetivas para estimar el desempeño individual y organizacional también constituye una limitación en la investigación realizada, ya que pueden estar sesgadas. Por último, también se resalta que los instrumentos diseñados para la medición de estos aspectos, al presentar un formato tipo encuesta y haber sido diseñados ad hoc para la investigación realizada, no cuentan con todas las estimaciones de validez requeridas de acuerdo con los standards internacionales.

En virtud de que se ha comprobado que las variables de tipo individual (e.g., sexo, edad) no presentan relación con los niveles de engagement de los trabajadores, cobra especial relevancia analizar en futuras investigaciones otras variables que puedan tener mayor influencia sobre este aspecto, como pueden ser los rasgos de personalidad, el comportamiento organizacional positivo o la pasión por el trabajo, conceptos que están siendo estudiados en el marco de la POP. Otro aspecto fundamental por indagar es la relación con las características de la organización y el tipo de tarea (Innstrand, 2016), lo cual sería de gran utilidad para el diseño de intervenciones organizacionales en busca de incrementar los niveles de engagement.

\section{REFERENCIAS}

Alarcon, G. \& Edwards, J. (2011). The Relationship of Engagement, Job Satisfaction and Turnover Intentions. Stress and Health Volume, 27(3), 294-298. https://doi.org/10.1002/smi.1365

Alarcon, G. \& Lyons, J. (2011). The Relationship of Engagement and Job Satisfaction in Working Samples. The Journal of Psychology, 145(5), 463-480. https://doi.org/10.1080/00223980.2011.584083

Albrecht, S. L., Bakker, A. B., Gruman, J. A., Macey, W. H., \& Saks, A. M. (2015). Employee engagement, human resource management practices and competitive advantage: An integrated approach. Journal of Organizational Effectiveness: People and Performance, 2(1), 7-35. https://doi.org/10.1108/JOEPP-08-2014-0042

Alessandri, G., Borgogni, L., Schaufeli, W., Caprara, G. V., \& Consiglio, C. (2015). From Positive Orientation to Job performance: The Role of Work Engagement and Self-efficacy Beliefs. Journal of Happiness Studies, 16(3), 767-788. https://doi.org/10.1007/s10902-014-9533-4

Bakker, A. B. \& Matthijs Bal, P. (2010). Weekly work engagement and performance: A study among starting teachers. Journal of Occupational and Organizational Psychology, 83, 189-206. https://doi.org/10.1348/096317909X402596

Bakker, A. B., Schaufeli, W. B., \& Leiter, M. P. (2008). Work engagement: An emerging concept in 
occupational health psychology. Work \& $\quad$ Stress, $22(3), \quad 187-200$. https://doi.org/10.1080/02678370802393649 Balzer, W. K., Kihm, J. A., Smith, P. C., Irwin, J. L., Bachiochi, P. D., Robie, C., Sinar, E. F., \& Parra, L. F.
(1997). User's manual for the Job Descriptive Index (JDI; 1997 Revision) and the Job in General (JIG) Scales. Bowling Green, OH: Bowling Green State University.

Cahill, K. E., McNamara, T. K., Pitt-Catsouphes, M., \& Valcour, M. (2015). Linking shifts in the national economy with changes in job satisfaction, employee engagement and work-life balance. Journal of Behavioral and Experimental Economics, 56, 40-54. https://doi.org/10.1016/j.socec.2015.03.002

Cameron, K. S., Brigth, D., \& Caza, A. (2004). Exploring the relationship between organizational virtuosness and performance. American Behavioral Scientist, 47(6), 1-24. https://doi.org/10.1177/0002764203260209

Cameron, K. S. \& Spreitzer, G. M. (2012). The Oxford Handbook of Positive Organizational Scholarship. New York: Oxford University Press.

Christian, M. S., Garza, A. S., \& Slaughter, J. E. (2011). Work Engagement: A Quantitative Review and Test Of Its Relations With Task and Contextual Performance. Personnel Psychology, 64, 89-136. https://doi.org/10.1111/j.1744-6570.2010.01203.x

Cohen, J. (1992). A power primer. Psychological Bulletin, 112(1), 155-159. https://doi.org/10.1037/00332909.112.1.155

Denecke, K. \& Nejdl, W. (2009). How valuable is medical social media data? Content analysis of the medical web. Information Sciences, 179(12), 1870-1880. https://doi.org/10.1016/j.ins.2009.01.025

Goštautaitè, B. \& Bučiūnienè, I. (2015). Work engagement during lifespan: the role of interaction outside the organization and task significance. Journal of Vocational Behavior, 89, 109-119. https://doi.org/10.1016/j.jvb.2015.05.001

Ho, V., Wong, S., \& Lee, C. H. (2011). A Tale of Passion: Linking Job Passion and Cognitive Engagement to Employee Work Performance. Journal of Management Studies, 48(1), 26-47. https://doi.org/10.1111/j.1467-6486.2009.00878.x

Høigaard, R., Giske, R., \& Sundsli, K. (2012). European Journal of Teacher Education, 35(3), 347-357. https://doi.org/10.1080/02619768.2011.633993

Hsieh, H. F. \& Shannon, S. E. (2005). Three approaches to qualitative content analysis. Qualitative Health Research, 15, 1277-1288. https://doi.org/10.1177/1049732305276687

Innstrand, S.T. (2016). Occupational Differences in Work Engagement: A Longitudinal Study Among Eight Occupational Groups in Norway. Scandinavian Journal of Psychology, 57(4), 338-349. https://doi.org/10.1111/sjop.12298

Kahn, W. A. (1990). Psychological conditions of personal engagement and disengagement at work. Academy of Management Journal, 33, 692-724. https://doi.org/10.5465/256287

Kahn, W. A. (1992). To be fully there: psychological presence at work. Human Relations, 45(4), 321-349. https://doi.org/10.1177/001872679204500402

Karatepe, O. M. \& Karadas, G. (2015). Do psychological capital and work engagement foster frontline employees' satisfaction? A study in the hotel industry. International Journal of Contemporary Hospitality Management, 27(6),1254-1278. https://doi.org/10.1108/IJCHM-01-2014-0028

Keyes, C. L., Hyson, S. J., \& Lupo, K. L. (2000). The Positive Organization: Leadership Legitimacy, Employee Well-Being, and the Bottom Line. The Psychologist-Manager, 4, 143-153. https://doi.org/10.1037/h0095888

Lin, W., Wang, L., Bamberger, P. A., Zhang, Q., Wang, H., Guo, W., Shi, J., \& Zhang, T. (2016). Leading future orientations for current effectiveness: The role of engagement and supervisor coaching in linking future work self-salience to job performance. Journal of Vocational Behavior, 92, 145-156. https://doi.org/10.1016/j.jvb.2015.12.002

Liu, J., Cho, S., \& Putra, E. D. (2017). The moderating effect of self-efficacy and gender on work engagement for restaurant employees in the United States. International Journal of Contemporary Hospitality Management, 29(1), 624-642. https://doi.org/10.1108/IJCHM-10-2015-0539

Lupano Perugini, de la Iglesia, G., Castro Solano, A., \& Fernández Liporace, M. (2017). Validation of a Work Engagement Scale. Profiles associated with high Performance and Job Satisfaction. Ciencias Psicologicas, 11(2), 127-137.

Mache S., Vitzthum K., Klapp B. F., \& Danzer, G. (2014) Surgeons' work engagement: influencing factors and relations to job and life satisfaction. Surgeon, 12(4), 181-190. https://doi.org/10.1016/j.surge.2013.11.015

Maslach, C., Schaufeli, W. B., \& Leiter, M. P. (2001). Job burnout. Annual Review of Psychology, 52, 397422. https://doi.org/10.1146/annurev.psych.52.1.397

Mastenbroek, N. J. J. M., Jaarsma, A. D. C., Demerouti, E., Muijtjens, A. M. M., Scherpbier, A. J. J. A., \& van Beukelen, P. (2014). Burnout and engagement, and its predictors in young veterinary professionals: the influence of gender. Veterinary Record, 174(6), 144-152. https://doi.org/10.1136/vr.101762

Matthijs Bal, P., De Cooman, R., \& Mol, S. T. (2013). Dynamics of psychological contracts with work engagement and turnover intention: The influence of organizational tenure. European Journal of Work and Organizational Psychology, 22(1), 107-122. https://doi.org/10.1080/1359432X.2011.626198

Mauno, S., Kinnunen, U., \& Ruokolainen, M. (2007). Job demands and resources as antecedents of work engagement: A longitudinal study. Journal of Vocational Behavior, 70(1), 149-171. https://doi.org/10.1016/j.jvb.2006.09.002

Nienaber, H. \& Martins, N. (2014). An Employee Engagement Instrument and Framework Building on Existing Research. Mediterranean Journal of Social Sciences, 5(20), 485-496. https://doi.org/10.5901/mjss.2014.v5n20p485

Owens, B. P., Baker, W. E., Sumpter, D. M., \& Cameron, K. S. (2016). Relational energy at work: 
Implications for job engagement and job performance. Journal of Applied Psychology, 101(1), 35-49. https://doi.org/10.1037/ap10000032

Rich, B. L., LePine, J. A., \& Crawford, E. R. (2010). Job Engagement: Antecedents and Effects On Job Performance. Academy of Management Journal, 53(3), 617-635. https://doi.org/10.5465/amj.2010.51468988

Rothbard, N. P. (2001). Enriching or depleting? The dynamics of engagement in work and family roles. Administrative Science Quarterly, 46, 655-84. https://doi.org/10.2307/3094827

Rothbard, N. P. \& Patil, S. V. (2012). Being There: Work Engagement and Positive Organizational Psychology. En K. S. Cameron \& G. M. Spreitzer (Eds.), The Oxford Handbook of Positive Organizational Scholarship (pp. 231-243). New York: Oxford University Press.

Salanova, M. \& Schaufeli, W. (2004). El engagement de los empleados: un reto emergente para la dirección de los recursos humanos. Estudios Financieros, 261, 109-134.

Schaufeli, W. B. \& Bakker, A. B. (2004). Job demands, job resources, and their relationship with burnout and engagement: A multi-sample study. Journal of Organizational Behavior, 25, 293-315. https://doi.org/10.1002/job.248

Seligman, M.E.P. (1999). The president's address. American Psychologist, 54, 559-532.

Spector, P.E. (1997). Job satisfaction: Application, assessment, causes and consequences. Thousand Oaks, CA: Sage.

Xu, J. \& Cooper Thomas, H. (2011). How can leaders achieve high employee engagement? Leadership \& Organization Development Journal, 32(4), 399-416. https://doi.org/10.1108/01437731111134661

Yakin, M. \& Erdil, O. (2012). Relationships Between Self-Efficacy and Work Engagement and the Effects on Job Satisfaction: A Survey on Certified Public. Social and Behavioral Sciences, 58, 370-378. https://doi.org/10.1016/j.sbspro.2012.09.1013

Zhang, Y., Rich, B. L., \& LePine, J.A. (2009, agosto). Transformational leadership and job performance: The mediating role of job engagement. Trabajo presentado en The annual meeting of the Academy of Management. Chicago, Estados Unidos.

Recibido 27-02-2018 | Aceptado 17-08-2018

Este trabajo se encuentra bajo una Licencia Creative Commons Atribución 4.0 Internacional que permite a terceros utilizar lo publicado siempre que se dé el crédito pertinente a los autores y a Psicodebate 